\title{
A replication study confirms the association of GWAS-identified SNPs at MICB and PLCE1 in Thai patients with dengue shock syndrome
}

\author{
Tran Ngoc Dang1', Izumi Naka', Areerat Sa-Ngasang², Surapee Anantapreecha², Sumalee Chanama², \\ Nuanjun Wichukchinda ${ }^{2}$, Pathom Sawanpanyalert ${ }^{3}$, Jintana Patarapotikul ${ }^{4}$, Naoyuki Tsuchiya ${ }^{1}$ and Jun Ohashi ${ }^{* *}$
}

\begin{abstract}
Background: Dengue shock syndrome (DSS), a severe life-threatening form of dengue infection, mostly occurs in children. A recent genome wide association study (GWAS) identified two SNPs, rs3132468 of major histocompatibility complex class I polypeptide-related sequence B (MICB) and rs3765524 of phospholipase C, epsilon 1 (PLCE1), associated with DSS in Vietnamese children. In this study, to examine whether an identical association is found in a different population, the association of these two SNPs with DSS was assessed in Thai children with dengue.

Methods: The rs3132468 and rs3765524 SNPs were genotyped in 917 Thai children with dengue: 76 patients with DSS and 841 patients with non-DSS. The allele frequencies were compared between DSS and non-DSS groups by one-sided Fisher's exact test. The association of rs3132468 and rs3765524 with the mRNA expression levels of MICB and PLCE1 were assessed in EBV-transformed lymphoblastoid cell lines.

Results: The reported DSS-risk alleles were significantly associated with DSS in Thai patients with dengue (one-sided $P=0.0213$ and odds ratio [OR] $=1.58$ for rs3132468-C and one-sided $P=0.0252$ and $\mathrm{OR}=1.49$ for rs3765524-C). The rs3132468-C allele showed a significant association with lower mRNA level of MICB $(P=0.0267)$, whereas the rs3765524-C allele did not. These results imply that the MICB molecule may play an important role in the prevention of DSS in dengue infection.
\end{abstract}

Conclusions: Together with previous association studies, we conclude that rs3132468-C at MICB and rs3765524-C at PLCE1 confer risk of DSS in Southeast Asians.

Keywords: Association, Dengue shock syndrome (DSS), Expression, MICB, PLCE1, Polymorphism

\section{Background}

Dengue is a mosquito-borne disease caused by the dengue virus, a small single-stranded RNA virus comprising four distinct serotypes. Approximately two fifths of the world's populations ( 2.5 billion people) are at risk of dengue, and Southeast Asia is one of the most seriously affected regions. The most severe form of dengue is dengue shock syndrome (DSS), a leading cause of death resulting from dengue infection [1].

The clinical outcome of DSS is affected by a number of factors $[2,3]$. Over the last decade, great efforts have been made to elucidate host genetic factors (i.e., polymorphisms)

\footnotetext{
* Correspondence: juno-tky@umin.ac.jp

${ }^{1}$ Faculty of Medicine, University of Tsukuba, Tsukuba, Japan

Full list of author information is available at the end of the article
}

that are involved in the severity of dengue [4,5]. Recently, a genome wide association study (GWAS) identified two loci, $\mathrm{MHC}$ class I polypeptide-related sequence B $(M I C B)$ and phospholipase $C$, epsilon 1 (PLCE1), associated with DSS in Vietnamese population [6]. Although GWAS provides strong evidence of association, replication studies in different populations or ethnic groups are required to examine whether the same association is commonly found in human populations regardless of the difference in environmental and genetic factors. Furthermore, in the case of infectious diseases, such as dengue, differences in the genetic background of infectious organisms may cause population-specific association. In the present study, in Thai patients with dengue, we investigated the association

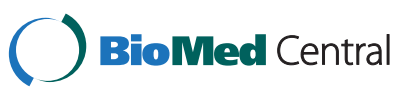

(c) 2014 Dang et al.; licensee BioMed Central Ltd. This is an Open Access article distributed under the terms of the Creative Commons Attribution License (http://creativecommons.org/licenses/by/2.0), which permits unrestricted use, distribution, and reproduction in any medium, provided the original work is properly credited. The Creative Commons Public Domain Dedication waiver (http://creativecommons.org/publicdomain/zero/1.0/) applies to the data made available in this article, unless otherwise stated. 
of two SNPs, rs3132468 and rs3765524, with DSS previously found in Vietnamese patients [6].

\section{Methods}

\section{Subjects}

A total of 917 patients with dengue, treated at Ratchaburi Hospital and Lampang Hospital, Thailand during 1999 to 2004, were examined in this study. All patients were $\leq 15$ years old at diagnosis. The dengue virus infection was confirmed in these patients by dengue IgM/IgG capture ELISA, RT-PCR, and/or dengue virus isolation at the Arbovirus Laboratory, National Institute of Health, Department of Medical Sciences, Ministry of Public Health, Thailand. The patients were classified into three groups: dengue fever (DF), dengue hemorrhagic fever (DHF) and DSS according to the WHO 1997 criteria [7]. DF is defined as an acute febrile stage with various nonspecific symptoms, such as headache, retro-orbital pain, myalgia, arthralgia, rash, hemorrhagic manifestations, leukopenia, and joint and muscular pain. DHF is diagnosed when the patient has additionally all the following symptoms: bleeding (hemorrhagic tendencies), plasma leakage $(\geq 20 \%$ change in hematocrit or signs of pleural effusion, ascites, and hypoproteinemia), and thrombocytopenia (platelet count $\leq 100,000 / \mathrm{mm}^{3}$ ). DSS, a severe form of dengue infection, is defined as DHF with tachycardia $(>100 /$ $\mathrm{min})$, narrow pulse pressure $(\leq 20 \mathrm{mmHg})$, or hypotension (systolic blood pressure $<90 \mathrm{mmHg}$ ) with cold clammy skin and restlessness.

This study was approved by the Institutional Review Board for Research on Human Subjects, the Department of Medical Science, Ministry of Public Health, Thailand; the Institutional Review Board of the Faculty of Tropical Medicine, Mahidol University, Thailand; and the Research Ethics Committee of the Faculty of Medicine, University of Tsukuba, Japan. Unlinked anonymous blood samples were obtained from the unidentifiable leftover blood of laboratory diagnosis of dengue infection. According to the guideline of Thailand [8], no specific consent forms were required from the patients in this case, since the Ethics Committee approved.

\section{Genotyping}

Genomic DNA was extracted from peripheral blood leukocytes using a QIAamp Blood Kit (Qiagen, Hilden, Germany). Two SNPs, rs3132468 at $M I C B$ and rs3765524 at PLCE1, were genotyped by TaqMan ${ }^{\circ}$ SNP Genotyping Assay (Applied Biosystems, Foster City, CA, USA).

\section{mRNA expression data}

Normalized mRNA data from Epstein-Barr virustransformed lymphoblastoid cell lines derived from 45 JPT and 45 CHB HapMap subjects were obtained from the database of the Gene Expression Variation (GENEVAR) project (http://www.sanger.ac.uk/resources/software/genevar/) $[9,10]$. The mRNA expression data detected by GI_26787987S probe for MICB and GI_19923454-S for PLCE1 were used in this study. The genotype data of rs3132468 and rs3765524 in HapMap JPT $+\mathrm{CHB}$ individuals were obtained from the HapMap database $[11,12]$.

\section{Statistical analyses}

To assess the association of rs3132468 and rs3765524 with DSS, the genotype and allele frequencies were compared by Fisher's exact test between DSS and non-DSS (DF + DHF). A logistic regression analysis adjusted for age, sex, and geographic location (i.e., hospital) was further performed to assess the association of each SNP with DSS. In a logistic regression analysis, the number of DSS-risk alleles (i.e., 0, 1, or 2) was used as an independent variable. Because the DSS-risk alleles (rs3132468-C and rs3765524-C) were previously identified [6], one-sided $P$-values were calculated. The associations of $M I C B$ and PLCE1 SNPs with mRNA expression levels of $M I C B$ and PLCE1 gene were evaluated, respectively, using a simple regression analysis with the number of DSS-risk allele as an independent variable. The significance level of a statistical test was set at 0.05 (one-sided $P$-value $<0.05$ and two-sided $P$-value $<0.05$ were considered to be statistically significant in association tests of DSS and mRNA expression level, respectively). To search for functional SNPs being in linkage disequilibrium (LD) with rs3132468 $\left(r^{2} \geq 0.8\right)$ in HapMap JPT and CHB populations [11,12], an in silico tool [13] was used. ESEfinder (http://rulai.cshl.edu/cgi-bin/tools/ESE3/esefinder.cgi? process=home) was used to assess whether SNPs are located in exonic splicing enhancers (ESEs). ESEs are binding sites for Ser/Arg-rich proteins (SR proteins) that have multiple functions in the pre-mRNA splicing process.

\section{Results}

\section{Patients with dengue}

A total of 917 laboratory-confirmed patients with dengue aged $\leq 15$ years old were investigated in this study. Patients were recruited from Ratchaburi Province and Lampang Province, Thailand. The patients with dengue were classified into three groups: 409 patients with DF, 432 patients with DHF, and 76 patients with DSS, according to WHO 1997 criteria (Table 1). In this study, patients with DF and DHF were regarded as patients with non-DSS.

\section{Association test}

The allele frequencies of rs3132468-C at MICB and rs3765524-C at PLCE1 were compared between DSS and non-DSS groups (Table 2). Because the significant associations of rs3132468-C and rs3765524-C with risk for DSS have been reported in Vietnamese children, the alternative hypothesis here should be that the allele frequencies of rs3132468-C at $M I C B$ and rs3765524-C at 
Table 1 Subjects investigated in this study

\begin{tabular}{|c|c|c|c|}
\hline \multirow[t]{2}{*}{ Characteristics } & \multicolumn{3}{|c|}{ Dengue patients } \\
\hline & $\mathrm{DF}(n=409)$ & DHF $(n=432)$ & DSS $(n=76)$ \\
\hline Age (years) ${ }^{a}$ & $9(1,15)$ & $10(0,15)$ & $9(3,15)$ \\
\hline \multicolumn{4}{|l|}{ Sex: } \\
\hline Male & 213 & 235 & 41 \\
\hline Female & 196 & 197 & 35 \\
\hline \multicolumn{4}{|l|}{ Region: } \\
\hline Ratchaburi & 137 & 322 & 52 \\
\hline Lampang & 172 & 110 & 24 \\
\hline \multicolumn{4}{|l|}{ Serotype: } \\
\hline DEN-1 & 153 & 136 & 15 \\
\hline DEN-2 & 109 & 158 & 39 \\
\hline DEN-3 & 92 & 73 & 6 \\
\hline DEN-4 & 54 & 64 & 16 \\
\hline DEN-3 + DEN-4 & 0 & 1 & 0 \\
\hline
\end{tabular}

${ }^{a}$ Median (minimum, maximum).

PLCE1 were increased in patients with DSS compared with patients with non-DSS. In other words, the onesided test should be conducted to confirm the previous finding. The one-sided Fisher's exact test showed that both reported DSS-risk alleles were associated with risk for DSS in Thai children (one-sided $P=0.0213$ and per allele odds ratio $[\mathrm{OR}]=1.58$ for $\mathrm{rs} 3132468-\mathrm{C}$; one-sided $P=0.0252$ and per allele $\mathrm{OR}=1.49$ for rs3765524-C).

To consider the effects of age, sex, and hospital (i.e., geographic location of subjects) on the association, a logistic regression analysis adjusted for these independent variables was further performed. The logistic regression analysis confirmed the significant association of rs3132468-C at MICB and rs3765524-C at PLCE1 with DSS in Thai patients with dengue (one-sided $P=0.0154$, per allele OR $=1.57$, two-sided 95\% CI: $1.11-2.22$ for rs3132468-C; one-sided $P=0.0226$, OR $=1.48$, two-sided 95\% CI: $1.07-$ 2.04 for rs3765524-C). The independent variables, age, sex, and geographic location, showed no significant association with DSS. The results from logistic regression analysis were consistent with those from the Fisher's exact test.

Association of DSS-risk allele with mRNA expression level To examine the functional significance of rs3132468 at $M I C B$ and rs3765524 at PLCE1, the association between the DSS-risk allele and the mRNA expression level was evaluated. The mRNA expression levels of $M I C B$ and PLCE1 in EBV-transformed lymphoblastoid cell lines from the HapMap JPT and CHB subjects are shown in Figure 1. The copy number of rs3132468-C in an individual (i.e., 0, 1 , or 2) was inversely associated with the level of mRNA expression of MICB ( $P=0.0267$ and partial regression coefficient $=-0.126)$, whereas no significant association was observed between rs3765524-C and mRNA level of PLCE1 $(P=0.0863$ and partial regression coefficient $=0.0209)$.

\section{In silico analysis}

The rs3132468 SNP is located in intron 6 of MICB. Although rs3132468 was statistically associated with risk for DSS, a SNP primarily associated with DSS may not be rs3132468. Therefore, we used an in silico tool [13] to search for functional SNPs being in LD with rs3132468. Seventeen SNPs were discovered to be in LD $\left(r^{2} \geq 0.8\right)$ with $r 33132468$ in HapMap JPT population (data not shown). One of these 17, rs3828916, in exon 2 of $M I C B$, was predicted by ESEfinder (http://exon.cshl. edu/ESE/) to be located in ESEs that are binding sites for SR proteins, SF2/ASF and SRp40. Because both alleles (i.e., rs3828916-C and rs3828916-G) demonstrated scores larger than the default threshold values (i.e., 1.956 for SF2/ASF and 2.670 for SRp40), it is unclear whether rs3828916 has functional significance. At the PLCE1 locus, a total of 28 SNPs were in LD $\left(r^{2} \geq 0.8\right)$ with rs3765524 in HapMap JPT + CHB populations. Of these 28 SNPs, rs753724, rs2274223, and rs11187870 were predicted to have a functional significance in the in silico analysis using the SNPinfo Web Server [13]. The rs753724 SNP located in the upstream region of PLCE1 was predicted to be located in the transcription factor binding sites. The rs2274223 is a nonsynonymous SNP, which may affect the PLCE1 protein function through amino acid alteration. The rs11187870 SNP was predicted to be located in microRNA-binding sites. Since the above mentioned SNPs may be primary ones with functional significance, we should not conclude that MICB rs3132468 and PLCE1 rs3765524 are causative SNPs associated with DSS.

\section{Discussion}

The present study revealed that $M I C B$ and PLCE1 SNPs are associated with a progression from less severe symptom of dengue to DSS in Thai children with dengue.

Table 2 Allelic association test for MICB rs3132468 and PLCE1 rs3765524

\begin{tabular}{llllrr}
\hline Allele (gene) & \multicolumn{2}{l}{ Dengue patients } & One-sided $P$-value & OR & 95\% Cl \\
\cline { 2 - 3 } & DSS & non-DSS & & 1.58 & $1.02-2.40$ \\
\hline rs3132468-C (MICB) & 0.217 & 0.149 & 0.0213 & 1.49 & $1.00-2.26$ \\
rs3765524-C (PLCE1) & 0.763 & 0.684 & 0.0252 & \\
\hline
\end{tabular}

$P$-value was calculated by using Fisher's exact test. 

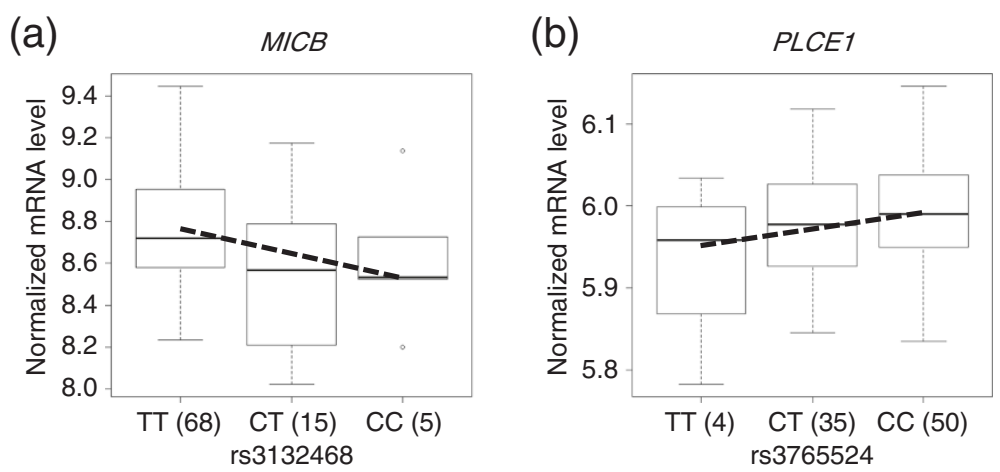

Figure 1 mRNA expression level and SNP genotype. (a) mRNA expression level of MICB in each genotype of rs3132468. The rs3132468-C allele was associated with lower mRNA expression level of MICB $(P=0.0267$ and partial regression coefficient $=-0.126)$. (b) mRNA expression level of PLCE1 in each genotype of rs3765524. The rs3765524-C allele was not associated with mRNA expression level of PLCE1 ( $P=0.0863$ and partial regression coefficient $=0.0209$ ). A regression line is represented by a dashed line. The number of samples for each genotype is presented in parentheses.

The roles of MICB and PLCE1 proteins in the pathogenesis of DSS remain to be elucidated. MICB is one of stress-induced molecules expressed by virus-infected cells. This molecule interacts with and activates the natural killer group 2 member D (NKG2D) receptor on NK cells. The activated NK cells induce the killing of the virus-infected cells. Our study showed that a DSS-risk allele, rs3132468-C of $M I C B$, was significantly associated with lower mRNA expression of $M I C B$. As suggested by Whitehorn et al. [14], dengue virus-infected cells with lower MICB expression are plausible to escape from the NKG2D-mediated killing by NK cells. Infected cells escaping the host immune response may mediate the pathogenesis of DSS because high dengue viremia titer is associated with increased disease severity [15]. This requires to be studied in future by the evaluation of the expression levels of MICB and the viremia titer in patients with dengue.

In this study, non-DSS group, compared with DSS group, consisted of patients with DF and DHF. When patients with DSS were compared only with patients with DF, the allele frequencies of rs3132468-C at MICB and rs3765524$C$ at $P L C E 1$ were significantly higher in DSS group than in DF group (one-sided $P=0.0119$ for rs3132468 at $M I C B$ and one-sided $P=0.0223$ for rs3765524 at PLCE1). When patients with DSS were compared only with patients with DHF, the difference in allele frequency of rs3765524-C at PLCE1 between DSS and DHF groups was statistically significant (one-sided $P=0.0399$ ), and the difference in allele frequency of rs3132468-C at MICB was marginally significant (one-sided $P=0.0521$ ). In contrast, no significant difference in allele frequencies of $\mathrm{rs} 3132468-\mathrm{C}$ and rs3765524-C was observed between DHF and DF groupss (one-sided $P=0.1454$ for rs3132468 at $M I C B$ and one-sided $P=0.3155$ for rs3765524 at PLCE1). These results suggest that rs3132468-C and rs3765524-C are not associated with risk for DHF except for DSS in Thai dengue patients.

Previous association studies using cord blood controls suggest that MICB and PLCE1 SNPs are associated with not only DSS [6] but also less severe form of dengue [14]. Our study design allows us to exclude the confounding factors involved in infection with dengue virus and symptomatic dengue infection. However, the limitation is that the possible association of MICB and PLCE1 SNPs with susceptibility to less severe form of dengue cannot be assessed, since only patients with symptomatic dengue infection are available. To examine this possibility in Thai dengue patients, patients with asymptomatic dengue infection remain to be investigated.

\section{Conclusions}

The present study in Thai patients with dengue successfully replicated the associations of $M I C B$ and PLCE1 SNPs with DSS that were reported by GWAS in Vietnamese patients [6]. Thus, we conclude that rs3132468$\mathrm{C}$ at MICB and rs3765524-C at PLCE1 are risk factors of DSS in Southeast Asians.

\section{Competing interests}

The authors declare that they have no competing interests.

\section{Authors' contributions}

TND and JO performed statistical analyses. TND and JO wrote the manuscript. AS and IN extracted DNA. TND performed genotyping. AS, SA, SC, NW, PS and JP collected blood samples and contributed to the acquisition of clinical data. TND, SA, NW, JP, and JO participated in the design and coordination of the study. NT was involved in the interpretation of the data and preparation of the manuscript. All authors read and approved the final manuscript.

\section{Acknowledgments}

We are deeply grateful to the patients who participated in this study. We are grateful to three reviewers for their valuable comments and suggestions. This work was partly supported by KAKENHI (25133702) Grant-in-Aid for 


\section{Author details}

${ }^{1}$ Faculty of Medicine, University of Tsukuba, Tsukuba, Japan. ${ }^{2}$ Department of Medical Sciences, Ministry of Public Health, Nonthaburi, Thailand. ${ }^{3}$ Food and drug Administration, Ministry of Public Heath, Nonthaburi, Thailand. ${ }^{4}$ Department of Microbiology and Immunology, Faculty of Tropical Medicine, Mahidol University, Bangkok, Thailand.

Received: 2 February 2014 Accepted: 12 May 2014

Published: 17 May 2014

\section{References}

1. Anders KL, Nguyet NM, Chau NV, Hung NT, Thuy TT, Lien le B, Farrar J, Wills $B$, Hien TT, Simmons CP: Epidemiological factors associated with dengue shock syndrome and mortality in hospitalized dengue patients in Ho Chi Minh City, Vietnam. Am J Trop Med Hyg 2011, 84:127-134.

2. Guzman MG, Halstead SB, Artsob H, Buchy P, Farrar J, Gubler DJ, Hunsperger E, Kroeger A, Margolis HS, Martinez E, Nathan MB, Pelegrino JL, Simmons C, Yoksan S, Peeling RW: Dengue: a continuing global threat. Nat Rev Microbiol 2010, 8:S7-S16.

3. Huy NT, Van Giang T, Thuy DH, Kikuchi M, Hien TT, Zamora J, Hirayama K: Factors associated with dengue shock syndrome: a systematic review and meta-analysis. PLoS Negl Trop Dis 2013, 7:e2412.

4. Lan NT, Hirayama K: Host genetic susceptibility to severe dengue infection. Trop Med Health 2011, 39:73-81.

5. Fang X, Hu Z, Shang W, Zhu J, Xu C, Rao X: Genetic polymorphisms of molecules involved in host immune response to dengue virus infection. FEMS Immunol Med Microbiol 2012, 66:134-146.

6. Khor CC, Chau TN, Pang J, Davila S, Long HT, Ong RT, Dunstan SJ, Wills B, Farrar J, Van Tram T, Gan TT, Binh NT, Tri le T, Lien le B, Tuan NM, Tham NT, Lanh MN, Nguyet NM, Hieu NT, Van NVinh Chau N, Thuy TT, Tan DE, Sakuntabhai A, Teo YY, Hibberd ML, Simmons CP: Genome-wide association study identifies susceptibility loci for dengue shock syndrome at MICB and PLCE1. Nat Genet 2011, 43:1139-1141.

7. Chow VT: Molecular diagnosis and epidemiology of dengue virus infection. Ann Acad Med Singapore 1997, 26:820-826.

8. Review of the Informed Consent. [http://www.tm.mahidol.ac.th/research/ Ethics/human/sop/ECS-006-04.pdf]

9. Yang TP, Beazley C, Montgomery SB, Dimas AS, Gutierrez-Arcelus M, Stranger BE, Deloukas P, Dermitzakis ET: Genevar: a database and Java application for the analysis and visualization of SNP-gene associations in eQTL studies. Bioinformatics 2010, 26:2474-2476.

10. Stranger BE, Nica AC, Forrest MS, Dimas A, Bird CP, Beazley $C$, Ingle $C E$, Dunning M, Flicek P, Koller D, Montgomery S, Tavare S, Deloukas P, Dermitzakis ET: Population genomics of human gene expression. Nat Genet 2007, 39:1217-1224.

11. The International_HapMap_Consortium: The International HapMap Project. Nature 2003, 426:789-796.

12. The International HapMap Consortium: A haplotype map of the human genome. Nature 2005, 437:1299-1320.

13. Xu Z, Taylor JA: SNPinfo: integrating GWAS and candidate gene information into functional SNP selection for genetic association studies. Nucleic Acids Res 2009, 37:W600-W605.

14. Whitehorn J, Chau TN, Nguyet NM, Kien DT, Quyen NT, Trung DT, Pang J, Wills B, Van Vinh Chau N, Farrar J, Hibberd ML, Khor CC, Simmons CP: Genetic variants of MICB and PLCE1 and associations with non-severe dengue. Plos One 2013, 8:e59067.

15. Vaughn DW, Green S, Kalayanarooj S, Innis BL, Nimmannitya S, Suntayakorn S, Endy TP, Raengsakulrach B, Rothman AL, Ennis FA, Nisalak A: Dengue viremia titer, antibody response pattern, and virus serotype correlate with disease severity. J Infect Dis 2000, 181:2-9.

doi:10.1186/1471-2350-15-58

Cite this article as: Dang et al:: A replication study confirms the association of GWAS-identified SNPs at MICB and PLCE1 in Thai patients with dengue shock syndrome. BMC Medical Genetics 2014 15:58.

\section{Submit your next manuscript to BioMed Central and take full advantage of:}

- Convenient online submission

- Thorough peer review

- No space constraints or color figure charges

- Immediate publication on acceptance

- Inclusion in PubMed, CAS, Scopus and Google Scholar

- Research which is freely available for redistribution 